\title{
Orientierende Röntgen`Kleinwinkelmessungen an Chloroplasten von Allium porrum
}

\author{
Von O. Kratky, W. Menke, A. Sekora, B. Paletta und M. Bischof \\ Aus dem Institut für physikalische Chemie der Universität Graz und dem Botanischen Institut der \\ Universität Köln \\ (Z. Naturforschg. 14 b, 307-311 [1959]; eingegangen am 11. Dezember 1958)
}

\begin{abstract}
Röntgen-Kleinwinkeluntersuchungen an verschiedenen Präparaten der Chloroplasten von Allium porrum haben ergeben:

1. Ein gefriergetrocknetes, lipidfreies Präparat zeigte eine diffuse Streuung mit typischem Blättchencharakter. Die Auswertung führt auf zwei Hauptanteile mit Blättchendicken von etwa 250 und 870 Å.

2. Angetrocknete pulverisierte Chloroplasten lassen einen B ragg schen Reflex bei 62 bis $63 \AA$ erkennen. Der starke allgemeine Streuuntergrund macht die Zahlenangaben etwas unsicher.

Es liegt nahe, Zusammenhänge zwischen der Periode von 63 Å und der Dicke isolierter Blättchen von $250 \AA$ A (nämlich der vierfachen) zu sehen.

3. Angetrocknete Chloroplasten, als gerollter Film aufgenommen, zeigen wieder einen B r a g g schen Wert von $63 \AA$, welcher einer senkrecht zur Lamellenebene stehenden Periode zuzuordnen ist. Dieses Präparat gibt noch zwei weitere Reflexe bei 42 und 48,5 Å.

4. Das isolierte Lipid ergibt ein Diagramm mit einem ausgeprägten Maximum bei $51 \AA \AA$.
\end{abstract}

Polarisationsoptische Untersuchungen hatten ergeben, daß Chloroplasten geschichtete Systeme darstellen, in welchen Proteinlamellen und LipoidDoppellamellen abwechseln ${ }^{1}$. Mit Hilfe des Elektronenmikroskopes konnte der lamellare Bau der Chloroplasten direkt sichtbar gemacht werden ${ }^{2}$. Die Deutung der feineren Einzelheiten elektronenmikroskopischer Aufnahmen ist jedoch unsicher und Größenmessungen mit dem Elektronenmikroskop sind an biologischen Objekten aus verschiedenen Gründen mit ganz erheblichen Fehlern behaftet ${ }^{3}$. Es liegt daher der Versuch nahe, einerseits nach RöntgenKleinwinkelreflexen zu suchen, welche die Periodizität dieses Systems in Richtung normal zu den Lamellen und in den in der Lamellenebene gelegenen Richtungen messen, und andererseits zu versuchen, aus der diffusen Röntgen-Kleinwinkelstreuung der möglichst in Einzellamellen zerlegten Chloroplasten auf die Dicke der Einzellamelle zu schließen. Der Versuch, Periodizitäten aufzufinden, wurde von FinEan, Sjöstrand und Steinmann ${ }^{4}$ unternommen. Sie betonen, daß die Kleinwinkelreflexe - offenbar wegen der kleinen Zahl von regelmäßig angeordneten beugenden Elementen - schlecht definiert sind. Sie berichten aber, an mit $\mathrm{OsO}_{4}$ fixierten Chloroplasten einen Reflex bei $250 \AA$ gefunden zu haben.

Eigene Versuche an nicht mit Schwermetallen behandelten Präparaten ergaben eine Mannigfaltigkeit

1 W. MENKE, Kolloid-Z. 85, 256 [1938].

2 W. MENKe, Protoplasma 35, 115 [1941] ; A. FreY-WyssLing u. K. Mühlethaler, Vjschr. naturforsch. Ges. Zürich 94, 179 [1949]; A. FreY-Wyssling, Suomen Kemistilehti A 29, 147 [1956]; J. J. Wolken u. G. E. Palade, Nature [London] 170, von Effekten und zwar konnten sowohl diffuse Streuungen als auch Streuungsmaxima aufgefunden werden. Die Breite dieser Reflexe ist allerdings so groß, $\mathrm{da} \beta$ schon die einwandfreie Bestimmung von B r a g g schen Werten Schwierigkeiten machte, weil die Abtrennung vom diffusen Untergrund mit Unsicherheiten behaftet ist. Immerhin dürfte es angebracht sein, über die Beobachtungen sowie die Auswertungen der diffusen Streuung kurz zu berichten, da sie den Ausgangspunkt für weitere Untersuchungen darstellen sollen.

\section{Herstellung der Präparate}

Blätter von Allium Porrum wurden mit der gleichen Menge 0,6-m. Saccharoselösung in einer Porzellanreibschale zerrieben. Aus dem durch Kolieren erhaltenen Extrakt wurden die Chloroplasten und andere größere Zellpartikel durch 30 Min. langes Zentrifugieren bei etwa $1500 \mathrm{~g}$ abzentrifugiert. Das Sediment wurde in wenig $0,6-m$. Saccharose verteilt und über einem Dichtegradienten aus Saccharoselösung eine Stde. bei $1500 \mathrm{~g}$ zentrifugiert. Diejenige Schicht, welche die Hauptmenge der Chloroplasten enthielt, wurde in dest. Wasser gegossen. Die Chloroplasten wurden abzentrifugiert und dreimal auf der Zentrifuge mit dest. Wasser gewaschen. Nach dem Abgießen des Überstandes wurden die Zentrifugengläser jedesmal einige Min. mit dem Sediment nach oben aufgestellt. Dabei fließen blasig angeschwollene Chloroplasten nach unten ab. Alle Aufarbeitungen erfolgten bei einer Raumtemperatur von $2^{\circ} \mathrm{C}$. Bei die-

114 [1952]; M. Cohn u. E. Bowler, Protoplasma 42, 414 [1953] ; H. Leyon, Exp. Cell. Res. 4, 371 [1953].

3 W. Menke, Z. Naturforschg. 12 b, 654, 656 [1957]; 13 b, 187 [1958].

4 J. B. Finean, F. S. Suöstrand u. E. Steinmann, Exp. Cell Res. 5, 557 [1953]. 
sem Arbeitsgang erhielt man Präparate, die bei mikroskopischer Kontrolle zu schätzungsweise $98 \%$ aus Chloroplasten bestanden, die keine feststellbaren Strukturstörungen aufwiesen. Aus ungeklärten Gründen erhielt man jedoch gelegentlich auch Präparate, in denen die Chloroplasten gänzlich in Lamellen und dünne Lamellenpakete zerfallen waren.

Ein solches Präparat, bestehend aus weitgehend zerfallenen Chloroplasten, wurde in flüssigen Stickstoff eingetropft und im gefrorenen Zustand über Kieselgel bei einem Druck von 0,1 Torr getrocknet. Dabei bleibt die Gestalt der Tropfen erhalten. Beim Berühren tritt ein Zerfall in ein sehr leichtes Pulver ein. Präparat 1 .

Ein Teil von Präparat 1 wurde bei Raumtemperatur erschöpfend mit peroxydfreiem Äther extrahiert und nach Abzentrifugieren im Vakuum von den $\ddot{\text { ththerresten }}$ befreit. Es verbleibt ein sehr lockeres weißes Pulver. Präparat 2 .

Ein wasserhaltiges Sediment, aus morphologisch weitgehend intakten Chloroplasten bestehend, wurde auf einer Glasplatte in möglichst gleichmäßig dünner Schicht ausgestrichen und über Kieselgel bei $20-30$ Torr getrocknet. Beim Antrocknen richten sich die Chloroplasten gegeneinander so aus, daß eine einachsig doppelbrechende Schicht entsteht, deren optische Achse normal zur Schichtebene austritt. Der trockene Film aus Chloroplasten wurde mit einer Rasierklinge abgehoben, wobei ein Pulver aus unregelmäßig begrenzten Blättchen entsteht. Präparat 3 .

Wurde eine auf einer Glasplatte angetrocknete Schicht von Chloroplasten kurze Zeit bei Raumtemperatur in ein mit Wasserdampf gesättigtes Gefäß gelegt, so ließ sich die Chloroplastenschicht als Film abheben. Dieser hat die Neigung sich aufzurollen. Präparat 4.

Zur Isolierung der Lipide wurde ein getrocknetes Chloroplasten-Präparat bei Raumtemperatur mit Methanol und anschließend mit Äther extrahiert. Die vereinigten Extrakte wurden im Vakuum bei $40^{\circ}$ Wasserbadtemperatur unter Stickstoff von den Lösungsmitteln befreit, der Rückstand mit trockenem, peroxydfreiem Äther aufgenommen, filtriert und der Äther durch Aufblasen von Stickstoff verjagt. Präparat 5.

\section{Röntgen-Kleinwinkeluntersuchung 5}

Zur Aufnahmetechnik sei erwähnt, daß die von dem einen von uns entwickelte blenden-streuungs-

5 Da das Verfahren nicht als allgemein bekannt angesehen werden darf, sei auf einige zusammenfassende Darstellungen verwiesen: A. Guinier u. G. Fournet, Small angle scattering of X-rays, Verlag J.Wiley \& Sons, Inc., New York, und Chapman u. Hall Ltd., London 1955; W. W. Beeman, P. Kaesberg, J. W. Anderegg u. M. B. Webb, Size of Particles and Lattice Defects, in: Handbuch der Physik, Band XXXII, herausgeg. von S. Fü̈GGE, Springer-Verlag, BerlinGöttingen-Heidelberg 1957; V. Gerold, Z. angew. Physik 9, 43 [1957]; O. Kratкx, Z. Elektrochem. Ber. Bunsenges. physik. Chem. 60, 245 [1956]; Naturwissenschaften 42, 237 [1955]. Der in der vorliegenden Arbeit angewendete freie Röntgen-Kleinwinkelkamera Anwendung fand ${ }^{6}$. Die hier diskutierten Aufnahmen waren mit einer durch Kristallreflexion monochromatisierten $\mathrm{CuK}_{\alpha^{-}}$ Strahlung hergestellt und zwar fand ein gebogener Kristall nach Johansson-Guinier ${ }^{7}$ Verwendung. Die Registrierung erfolgte mittels des photographischen Films und nachfolgender Photometrierung.

Bei den meisten Aufnahmen wurde ein spaltförmiger Primärstrahl verwendet. Soweit eine diffuse Streuung ausgewertet werden sollte, waren die Aufnahmen daher zur Beseitigung des Kollimationsfehlers nach bekanntem Verfahren ${ }^{8}$ zu entschmieren. Bei orientierten Präparaten, die bevorzugt in Richtung normal zum Primärstrahl streuen, entfällt die Entschmierung, ebenso bei Aufnahmen, wo der Primärstrahl genügend kurz gewählt wurde.

Nun sollen die an den fünf oben beschriebenen Präparaten erhaltenen Ergebnisse kurz besprochen werden.

Präparat 1: Gefriergetrocknet, lipidhaltig. Es wurde eine sehr steil ansteigende diffuse Kleinwinkelstreuung erhalten, die auf Clusterbildung schließen läßt. Eine quantitative Auswertung haben wir nicht versucht.

Präparat 2: Gefriergetrocknet, lipidfrei. Es war zu hoffen, daß an diesen Präparaten die Clusterbildung schwächer ist. Die immer noch sehr steil an-

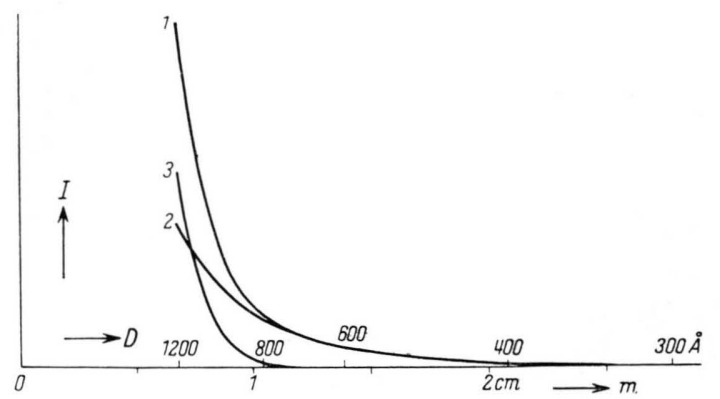

Abb. 1. Präparat 2: Die Gesamtstreukurve 1 ist additiv aus den Teilstreukurven 2 und 3 zusammensetzbar (vgl. Abb. 2) $a=21^{\circ} 2 \mathrm{~cm}, p=25^{\circ} 4$.

Auswertungsgang ist insbesondere aus den beiden letztgenannten Arbeiten verständlich; dort weitere Literatur.

${ }^{6}$ O. Kratky, Z. Elektrochem. Ber. Bunsenges. physik. Chem. 58, 49 [1954] ; 62, 66 [1958] ; Kolloid-Z. 144, 110 [1955]; O. Kratky u. A. Sekora, Mh. Chem. 85, 660 [1954]; O. Kratky u. Z. Skala, Z. Elektrochem. Ber. Bunsenges. physik. Chem. 62, 73 [1958].

7 T. Johansson, Z. Physik. 82, 587 [1933] ; A. Guinier, C. R. hebd. Séances Acad. Sci. 223, 31 [1946].

8 A. Guinier u. G. Fournet, J. phys. rad. 8, 345 [1947]; J. M. W. Du Mond, Physic. Rev. 72, 83 [1947]; O. Kratky, G. Porod u. L. Kahovec, Z. Elektrochem. Ber. Bunsenges. physik. Chem. 55, 53 [1951]. 
steigende Streukurve machte die Verwendung von Zeitserien (Belichtungszeiten von 1,5 Min. bis 18 Stdn.) erforderlich. Die Aufnahmen konnten durch die den verschiedenen Belichtungszeiten entsprechende Umrechnung der Schwärzungen zusammengefügt und entschmiert werden (Abb. 1). Von der Idee ausgehend, daß Blättchen vorliegen müssen, wurde der Dickenfaktor in der G u i n i e r schen Auftragung dargestellt, d. h. $\log \left(I \mathrm{~m}^{2}\right)$ gegen $\mathrm{m}^{2}$ aufgetragen*. Wir wir aus Abb. 2 ersehen, besteht

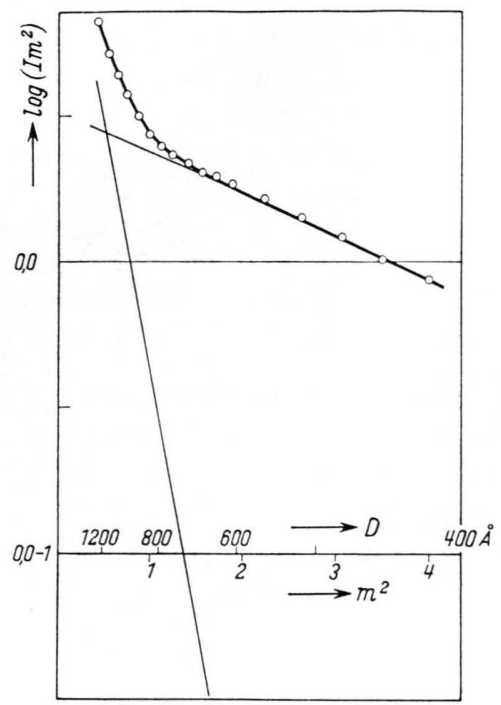

Abb. 2. Präparat 2: Auftragung des Dickenfaktors $I \mathrm{~m}^{2}$ im Sinne von G u i n i e r. Der gesamte Dickenfaktor läßt sich in zwei Teilstreukurven zerlegen, die nahezu geradlinig verlaufen. Nur im Knick weicht die durch Superposition der geradlinigen Teilkurven erhaltene Kurve (ausgezogene Linie) von der durch die eingezeichneten Punkte festgelegten experimentellen Kurve ein wenig ab. $a=21^{\circ} 2 \mathrm{~cm}, p=25^{\circ} 4$. Die Zusammensetzung in der Darstellung von $I$ gegen $m$ zeigt Abb. 1 .

diese Kurve aus zwei genähert geradlinigen Ästen, so daß sich der Versuch der Zerlegung in zwei Teile empfiehlt. Durch Probieren konnte gezeigt werden, daß der gemessene Verlauf tatsächlich durch Überlagerung von zwei Geraden (in der $\mathrm{Guinierschen}$ Darstellung) befriedigend möglich ist, die Dickenwerte von $250 \AA$ (äußerer Teil) und $870 \AA$ (innerer Teil) ergeben. Abb. 1 läßt die Zerlegung in der normalen Auftragung von $I$ gegen $m$ erkennen. Trotz dieser in formaler Hinsicht befriedigend scheinenden Interpretation ist hinsichtlich der Aussagen über die

* I ist die gemessene Intensität, $m$ der auf der Photometerkurve gemessene Abstand von der Primärstrahlmitte in $\mathrm{cm}$. Er hängt mit dem Ablenkungswinkel $2 \vartheta$ nach der Beziehung zusammen:

$$
2 \vartheta=m / a p,
$$

tatsächlichen Dicken dennoch Zurückhaltung geboten. Ganz offenkundig liegt eine Mischstreukurve vor, deren Auswertung kaum eindeutig erfolgen kann. Immerhin ist der theoretisch zu erwartende Typus der Blättchenkurve überzeugend realisiert, und es wird wohl auch der aus dem äußeren Teil entnommene Wert von $250 \AA$ der tatsächlichen Dicke von auftretenden Lamellen nahekommen. Bemerkenswerterweise besteht vollständige Übereinstimmung mit dem erwähnten Wert, den Finean, Sjöstrand und Steinmann ${ }^{4}$ an Präparaten erhalten haben, die mit $\mathrm{OsO}_{4}$ fixiert waren. Über die innere Struktur der Lamellen ist aus diesen Aufnahmen natürlich nichts zu entnehmen. Der innere Teil der Kurve läßt erkennen, daß auch Cluster der mehrfachen Dicke vorliegen. Noch weiter innen steigt die Kurve noch steiler an, was auf eine Mannigfaltigkeit von Komplexen schließen läßt.

Die Streukurve wurde auch in doppeltlogarithmischer Auftragung dargestellt (Abb. 3). Es ergibt sich im Auslauf eine Neigungstangente von minus 3,9 in guter Übereinstimmung mit dem nach der Theorie

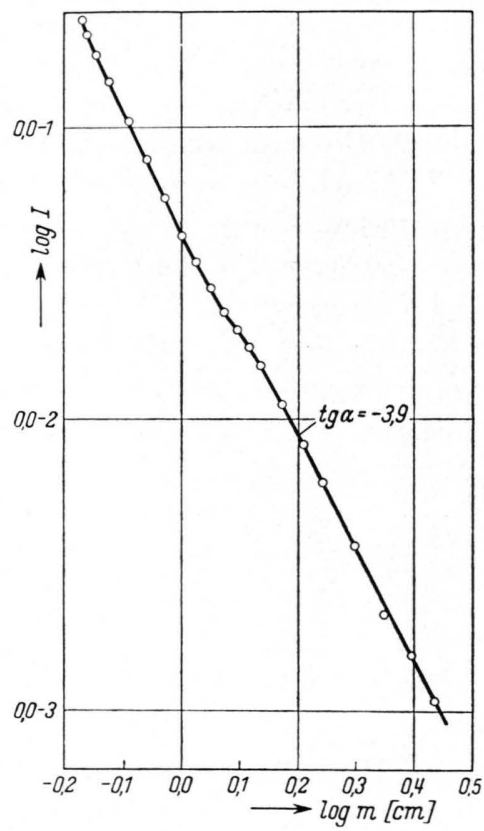

Abb. 3. Präparat 2: Auslauf der Streukurve in doppeltlogarithmischer Darstellung. Die Neigungstangente im Auslauf kommt dem nach $\mathrm{P}$ o r o d zu erwartenden theoretischen Wert von -4 sehr nahe. $a=21^{\circ} 2 \mathrm{~cm}, p=25^{\circ} 4$.

wo $a$ den Abstand Präparat-Film, $p$ das Ubersetzungsverhältnis der Photometerkurve darstellt.

Auf der Abszisse wird stets auch die Skala der rein formal nach der B rag g schen Beziehung berechneten Netzebenenabstände $D$ angebracht. 
von Porod ${ }^{9}$ zu erwartenden Verhalten, daß im Auslauf eines zweiphasigen regellosen Systems eine Neigungstangente von minus 4 auftreten müsse. Die Intensität dieses Auslaufes gestattet eine Berechnung der inneren Oberfläche. Es ergab sich, daß der erhaltene Wert um mehr als eine Größenordnung unter dem auf den Lamellendicken von $250 \AA$ A berechneten lag, woraus zu schließen ist, daß nur ein verhältnismäßig kleiner Teil der Substanz in Form von Einzellamellen vorlag.

Der einzige Weg, hier weiterzukommen, besteht wohl in einer Variation der Präparierungs-Bedin-

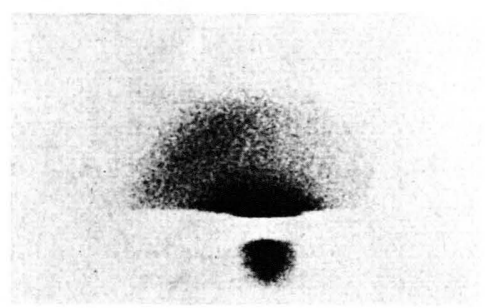

Abb. 4. Präparat 3: Durch das Einfüllen des Pulvers in die Kapillare tritt eine Orientierung ein, wodurch die Interferenz bei $62-63 \AA \AA$ als Sichel auftritt.

gungen. Immerhin erweist sich auch hier die Röntgen-Kleinwinkelstreuung als ein Weg, neben dem Gestaltstypus die Dimensionen zu ermitteln.

Präparat 3: Angetrocknete Chloroplasten, pulverisiert. Das Antrocknenlassen verfolgte das Ziel, eine Schichtung zu erzeugen, die die Größe der Periode direkt als Bragg scher Reflex erkennen lassen müßte. Bei Aufnahmen, bei welchen die Präparatachse (Achse der Kapillare) senkrecht zur Ebene des Primärstrahls stand, traten in der Nähe des Meri-

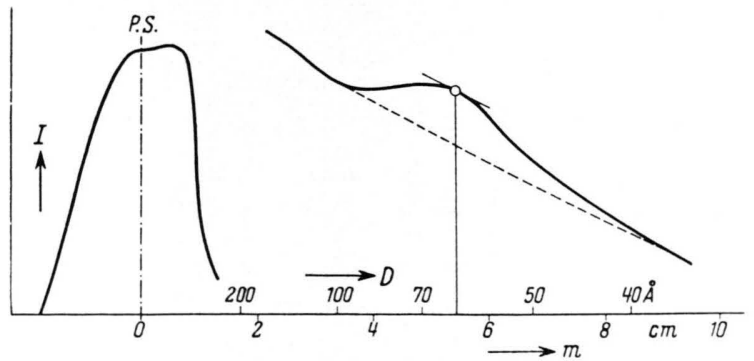

Abb. 5. Präparat 3: Umzeichnung der Photometerkurve von Abb. 4. $a=8^{\circ} 65 \mathrm{~cm}, p=25^{\circ} 4$.

dians Interferenzsicheln auf. Das bedeutet, daß sich die Lamellen offenbar durch das Einfüllen in die Kapillare orientiert hatten. Normalerweise wird man

${ }^{9}$ G. Porod, Kolloid-Z. 124, 83 [1951]; 125, 51 [1951]. erwarten, daß die Lamellenebene senkrecht zur Achse steht, also der Meridianreflex die auf die Lamellendicke zurückgehende Periode eines Schichtsystems mißt. Allerdings scheint die Orientierung von unkon-

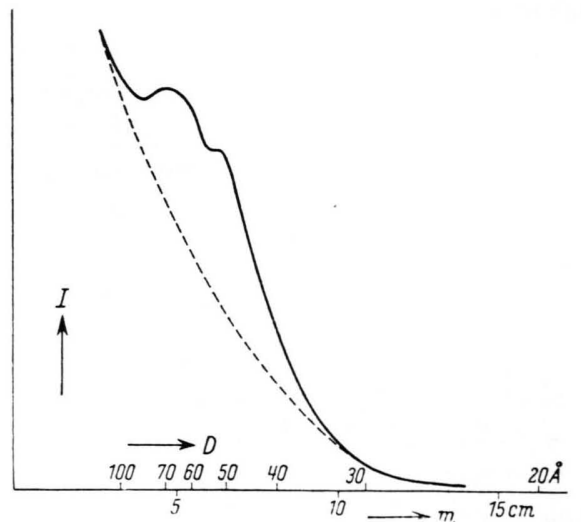

Abb. 6. Präparat 4: Umzeichnung der Photometerkurve mit eingezeichnetem Untergrund. $a=20^{\circ} 7 \mathrm{~cm}, p=10^{\circ} 2$.

trollierbaren Zufällen beeinflußt $\mathrm{zu}$ werden. Wie Abb. 4 zeigt, war z. B. eine besonders deutliche sichelförmige Interferenz erhalten worden, deren Maximum um etwa $25^{\cup}$ vom Meridian entfernt war. Die Auswertung führt auf einen B r a g g schen Netzebenenabstand von 62 bis $63 \AA$, wenn man den

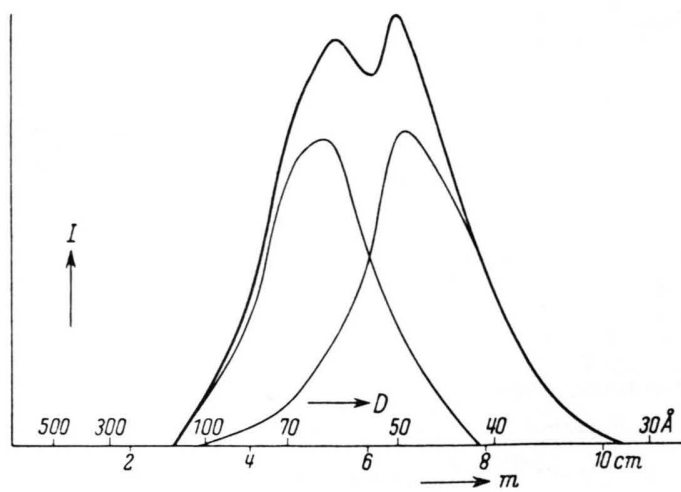

Abb. 7. Präparat 4: Versuch einer Zerlegung des über dem Untergrund in Abb. 6 befindlichen Streuanteils in zwei Maxima.

Untergrund so legt, wie das die Umzeichnung Abb. 5 der Photometerkurve erkennen läßt.

Präparat 4: Angetrocknete Chloroplasten, zu Röllchen geformt. Die Durchleuchtung bei Einstellung der Präparatachse parallel zur Strahlenebene ergab zwei mit freiem Auge erkennbare Reflexe. In der Photometerkurve fließen die beiden Maxima allerdings zusammen und die Zerlegung ist nicht willkürfrei. Trennt man den Untergrund so $a b$, wie das 
Abb. 6 zeigt, und nimmt man dann die Zerlegung des über dem Untergrund befindlichen Teiles gemäß Abb. 7 vor, so werden die B r a g g schen Werte von etwa 62,5 und $48,5 \AA$ erhalten. Stellt man die Präparatachse aber senkrecht zur Ebene des Strahls, so findet man einen Reflex, dessen Maximum bei Annahme des Untergrundes gemäß Abb. 8 bei 41,5 bis $42 \AA$ liegt.

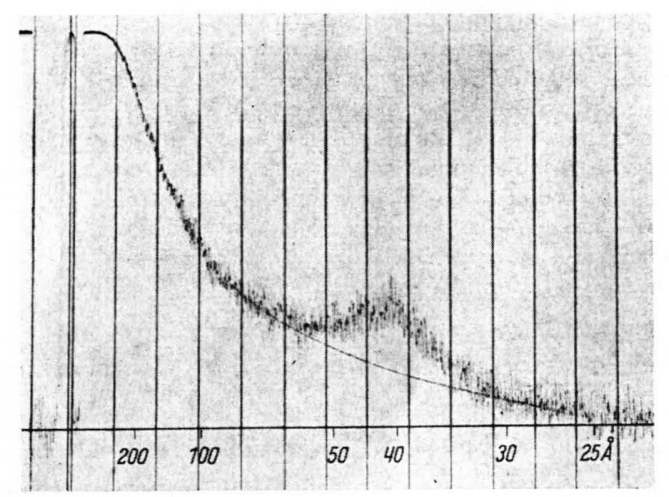

Abb. 8. Präparat 4: Photometerkurve des Röllchens bei Einstellung der Präparatachse normal zur Strahlenebene.

Nach dem Gesagten ist es kaum zweifelhaft, daß der Reflex von 62 bis $63 \AA$ mit der Lamellendicke zusammenhängt, also offenbar eine höhere Ordnung der Gesamtdicke darstellt. Er tritt nämlich immer am Meridian auf, wenn die Lamellenebene jedenfalls eines Teiles der Chloroplasten in der Strahlenebene liegt. Ob der 48-Å-Reflex zur gleichen Periode gehört, läßt sich nicht mit Sicherheit sagen, da er wohl beim gerollten Film auftritt, nicht aber bei den offenbar ziemlich gut orientierten Pulvern. Dies würde dafür sprechen, daß er eine in der Filmebene liegende Periode darstellt. Das gleiche ist für den
42-Ä-Reflex zu sagen, der seinen Ursprung in einer parallel zur Filmachse verlaufenden Periode hat. Diese Deutungen sind immerhin nur vorläufiger Natur, ihre Überprüfung mit Präparaten sehr genau definierter Orientierung bei Durchleuchtung in mindestens drei aufeinander normalen Richtungen ist notwendig.

Präparat 5: Das isolierte Lipid. Zur Kontrolle, ob einer der Reflexe vielleicht auf selbständige Kristallisation des Lipids zurückzuführen ist, wurde dieses durch Extraktion in reiner Form dargestellt und für sich untersucht. Die erhaltene Streukurve mußte entschmiert werden, weil das Präparat ungeordnet ist. Wir erhalten dann den in Abb. 9 dargestellten Streuverlauf mit einem sehr ausgeprägten Maximum bei $51 \AA$. Es scheint danach keiner der an den Chloroplasten-Präparaten beobachteten Reflexe auf das selbständig kristallisierte Lipid zurückzuführen zu sein.

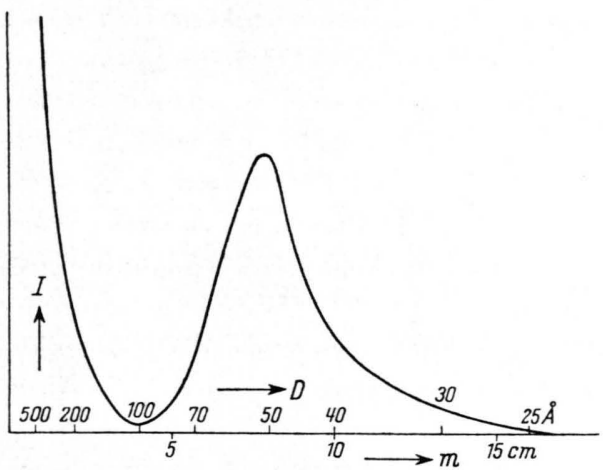

Abb. 9. Präparat 5: Die Streukurve des reinen Lipids, nach Entschmierung. $a=10^{\circ} 2 \mathrm{~cm}, p=25^{\circ} 4$.

Der eine Autor (Menke) dankt der De u t s chen Forschungsgemeinschaft für ihre Unterstützung. 